\title{
Superconductivity in plutonium compounds
}

\author{
J.L. Sarrao, E.D. Bauer, J.N. Mitchell, P.H. Tobash, and J.D. Thompson \\ Los Alamos National Laboratory, Los Alamos, NM 87545
}

\begin{abstract}
Although the family of plutonium-based superconductors is relatively small, consisting of four compounds all of which crystallize in the tetragonal HoCoGa $\mathrm{H}_{5}$ structure, these materials serve as an important bridge between the known Ce- and U-based heavy fermion superconductors and the high-temperature cuprate superconductors. Further, the partial localization of $5 \mathrm{f}$ electrons that characterizes the novel electronic properties of elemental plutonium appears to be central to the relatively high superconducting transition temperatures that are observed in $\mathrm{PuCoGa}_{5}, \mathrm{PuRhGa}_{5}, \mathrm{PuCoIn}_{5}$, and $\mathrm{PuRhIn}_{5}$.
\end{abstract}

Keywords:

plutonium, superconductivity, $\mathrm{PuCoGa} 5$

\section{Introduction}

$\mathrm{PuCoGa}_{5}$ was the first plutonium-based superconductor, and it displayed the rather high transition temperature, $\mathrm{T}_{c}$, of $18.5 \mathrm{~K}[1]$. Although this discovery was rather surprising, the initial perspective that $\mathrm{PuCoGa}_{5}$ was related to the previously discovered Ce-115 $\left(\mathrm{CeCoIn}_{5}, \mathrm{CeRhIn}_{5}\right.$, and $\left.\mathrm{CeIrIn}_{5}\right)$ superconductors and reflected the novel properties of elemental plutonium has survived the test of time $[2,3,4]$. In fact, the subsequent discoveries of superconductivity in $\mathrm{PuRhGa}_{5}, \mathrm{PuCoIn}_{5}$, and $\mathrm{PuRhIn}_{5}$ and their respective physical properties further confirm this picture.

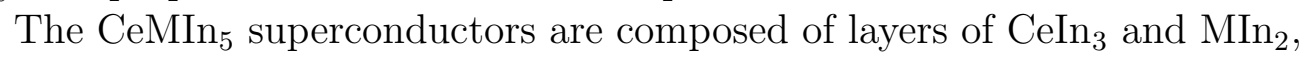
and much of the physics of these materials can be described by considering the materials as layered variants of $\mathrm{CeIn}_{3}[5,2,6]$. In fact, the Ce-115

*email: sarrao@lanl.gov, phone: +1-505-667-6645 
family of materials includes examples that have single layers of $\mathrm{CeIn}_{3}$ separated by bilayers of $\mathrm{MIn}_{2}$ (e.g., superconducting $\mathrm{CePt}_{2} \mathrm{In}_{7}$ [7]) as well as bilayers of $\mathrm{CeIn}_{3}$ separated by single layers of $\mathrm{MIn}_{2}$ (e.g., $\mathrm{Ce}_{2} \mathrm{RhIn}_{8}$, which displays pressure-induced superconductivity [8]). Pursuing this metaphor, $\mathrm{PuCoGa}_{5}$ would be described as made up of layers of $\mathrm{PuGa}_{3}$ and $\mathrm{CoGa}_{2}$. Further, $\mathrm{PuGa}_{3}$ is directly related to the high-temperature, face-centeredcubic (fcc) $\delta$-phase of $\mathrm{Pu}$, which is stabilized to room temperature by the addition of small amounts of Ga. Continuing to add $\mathrm{Ga}$ to elemental $\mathrm{Pu}$ leads one to the binary compound $\mathrm{Pu}_{3} \mathrm{Ga}$ and, at least figuratively, to fcc $\mathrm{PuGa}_{3}$ (in reality, $\mathrm{PuGa}_{3}$ crystallizes in either a hexagonal or rhombohedral structure [9]), the parent compound and analog of $\mathrm{CeIn}_{3}$ for $\mathrm{PuCoGa}_{5}$. Figure 1 depicts the $\mathrm{Pu}-115$ related crystal structures that have been synthesized to date[10, 13]. Following the discovery of $\mathrm{PuCoGa}_{5}, \mathrm{PuRhGa}_{5}\left(\mathrm{~T}_{c}=8.6 \mathrm{~K}\right)$ [11], $\mathrm{PuCoIn}_{5}\left(\mathrm{~T}_{c}=2.5 \mathrm{~K}\right)[12]$, and $\mathrm{PuRhIn}_{5}\left(\mathrm{~T}_{c}=1.7 \mathrm{~K}\right)[3]$ have also been reported and have been shown to be superconducting. Pu-based 1-2-7 and 2-1-8 compounds have also been reported (e.g., $\mathrm{Pu}_{2} \mathrm{PtGa}_{8}$ and $\mathrm{PuPt}_{2} \mathrm{In}_{7}$, but superconductivity has yet to be observed in these structures [13].

Plutonium is a fascinating metal. Its $5 \mathrm{f}$ electrons straddle the boundary between localized and itinerant behavior. The low-temperature, monoclinic $\alpha$-Pu displays itinerant f-electron behavior, while the higher-temperature $\delta$ $\mathrm{Pu}$ phase displays partially localized f-electron behavior, which, as noted above, can be stabilized to room temperature by the addition of small amounts of impurities such as Ga. This easily perturbed electronic configuration gives rise to an extremely complex metallurgy for plutonium metal[14] and challenges the state of the art in electronic structure calculation methods [15]. The crossover from localized to itinerant f-electron behavior is central to the phenomenology of the broader family of heavy fermion compounds [16] and is also reflective of unconventional superconductors that exist near magnetically ordered phases, as occurs in the Ce-115 compounds [6].

\section{Synthesis and Structural Properties}

Although relatively few groups can actively work with plutonium because of its radiological hazards, large single crystals of each of the known plutonium superconductors have been grown using molten metal (Ga or In) flux techniques, and single-crystal structural determinations have also been made for each compound. Pictures of representative crystals of these materials are shown in Figure 2 [13]. The structural and ground state properties of these 


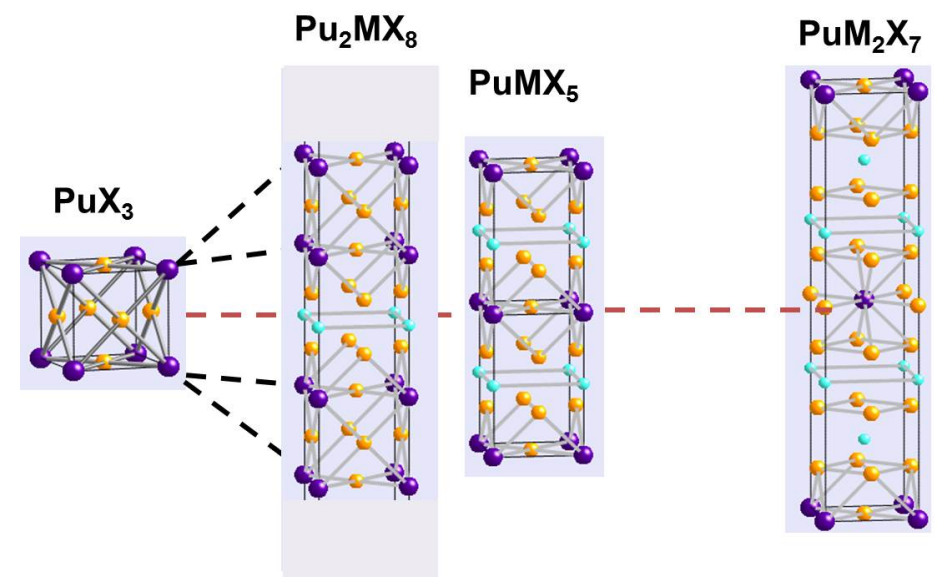

Figure 1: An illustration of the stacking orders that give rise to the $\mathrm{Pu}-115, \mathrm{Pu}-218$, and $\mathrm{Pu}-127$ stoichiometries. $\mathrm{M}$ is a transtion metal, and $\mathrm{X}$ can be Ga or In.

materials are summarized in Table 1. Full structural details of the Pu-based superconductors have been reported by Bauer et al. [13].

As will be discussed below, an interesting feature of the $\mathrm{Pu}-115$ superconductors (as well as the Ce-115 superconductors) is the linear correlation between $\mathrm{T}_{c}$ and the ratio of the tetragonal lattice constants, c/a, independent of chemical composition [17]. It thus appears that the spacing between alternating layers of $\mathrm{PuX}_{3}(\mathrm{X}=\mathrm{In}$ or $\mathrm{Ga})$ and $\mathrm{MX}_{2}(\mathrm{M}=\mathrm{Co}$ or $\mathrm{Rh})$ is directly correlated with superconducting transition temperature in a way that no other independent structural parameter is. Similarly, intermediate concentrations of $\mathrm{Rh}$ in $\mathrm{PuCo}_{1-x} \mathrm{Rh}_{x} \mathrm{Ga}_{5}$ display the same behavior, indicating that layer spacing is an essential parameter while, in contrast to many other families of superconductors, Rh-Co disorder in the $(\mathrm{Rh}, \mathrm{Co}) \mathrm{Ga}_{2}$ layer does not have a deleterious effect on superconducting transition temperature. This is the first of several indications that the superconductivity in $\mathrm{Pu}-115$ materials is qausi-two-dimensional and driven by the behavior of the $\mathrm{PuGa}_{3}\left(\mathrm{PuIn}_{3}\right)$ layer, both of which display long-range magnetic order as compounds.

\section{Superconducting Properties}

Bulk superconductivity in $\mathrm{PuCoGa}_{5}, \mathrm{PuRhGa}_{5}$, and $\mathrm{PuCoIn}_{5}$ has been demonstrated by heat capacity measurements $[1,18,12]$. Further, the jump $\Delta \mathrm{C}$ in specific heat at $\mathrm{T}_{c}$ allows an estimate of the Sommerfeld coefficient 


\begin{tabular}{|c|c|c|c|c|}
\hline & $\mathrm{PuCoGa}_{5}$ & $\mathrm{PuRhGa}_{5}$ & $\mathrm{PuCoIn}_{5}$ & $\mathrm{PuRhIn}_{5}$ \\
\hline $\mathrm{T}_{c}(\mathrm{~K})$ & 18.5 & 8.6 & 2.5 & 1.7 \\
\hline Lattice Constant $(A)$ & $\mathrm{a}=4.232$ & $\mathrm{a}=4.301$ & $\mathrm{a}=4.574$ & $\mathrm{a}=4.621$ \\
& $\mathrm{c}=6.786$ & $\mathrm{c}=6.857$ & $\mathrm{c}=7.439$ & $\mathrm{c}=7.460$ \\
\hline Discovery & Sarrao, 2002 $[1]$ & Wastin, 2003 $[11]$ & Bauer, 2012 [12] & Bauer, 2012 [3] \\
\hline
\end{tabular}

Table 1: Superconducting transition temperature, lattice constants, and original reference for known plutonium supercondcutors
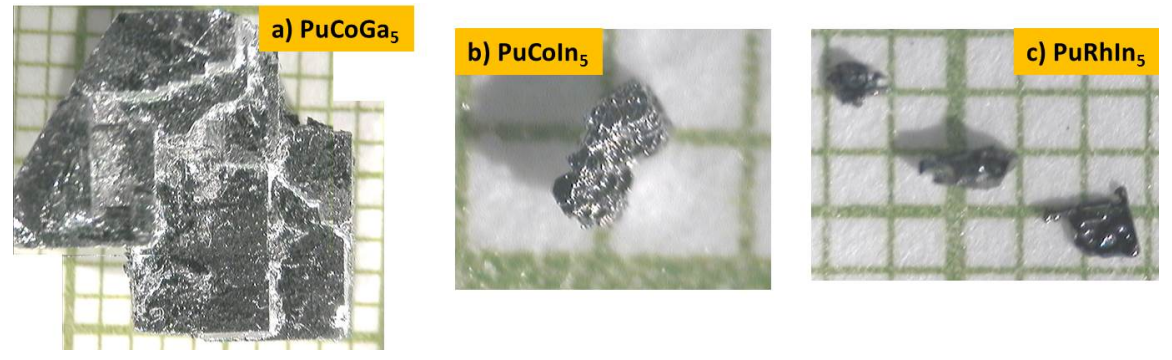

Figure 2: Pictures of representative crystals of known plutonium superconductors. The grid lines in the images are a millimeter scale.

(assuming the BCS relation $\Delta \mathrm{C} / \gamma \mathrm{T}_{c}=1.43$ ) for $\mathrm{PuCoIn}_{5}, \mathrm{PuCoGa}_{5}$, and $\mathrm{PuRhGa}_{5}$ of 150,100 , and $50 \mathrm{~mJ} / \mathrm{mol} \mathrm{K}{ }^{2}$, respectively, indicating heavy fermion behavior. The inferred values of the Sommerfeld coefficient are also consistent with the value of $\mathrm{C} / \mathrm{T}$ at temperatures just about $\mathrm{T}_{c}$ given in Table 2 [3]. Although superconductivity in $\mathrm{PuRhIn}_{5}$ has not yet been observed by heat capacity measurements due to its low $\mathrm{T}_{c}$, thermal expansion as well as resistivity, susceptibility, and NMR measurements indicates bulk, heavy fermion superconductivity in $\mathrm{PuRhIn}_{5}[13]$.

An interesting feature of the $\mathrm{Pu}-115$ superconductors is that, due to selfirradiation from radioactive $\mathrm{Pu}$, the superconducting transition temperature decreases as a function of time [19]. This has been studied most carefully in $\mathrm{PuCoGa}_{5}$ and $\mathrm{PuRhGa}_{5}$ because of their relatively higher transition temperatures [20]. The damage created by self-irradiation also produces pinning centers, which cause the upper critical field to increase as a function of time [20]. Jutier et al. [21] have used such data to analyze the nature of the superconducting pairing in $\mathrm{PuCoGa}_{5}$, suggesting a phononic mechanism in contrast to the more common 'dirty d-wave'picture. On the other hand, estimates of the electron-phonon coupling via measurements of the ultrafast 
optical response in $\mathrm{PuCoGa}_{5}$ argue against a phonon-based mechanism[22]

The upper critical field $H_{c 2}(0)$ as well as the initial temperature derivative of $H_{c 2}(T), \partial H_{c 2} / \partial T @ \mathrm{~T}_{c}$ for $\mathrm{PuMX}_{5}$ is included in Table 2 [3, 23, 24]. For both $\mathrm{PuCoIn}_{5}\left(H_{c 2}^{c}(0)=10 \mathrm{~T}\right)$ and $\mathrm{PuRhIn}_{5}\left(H_{c 2}^{c}(0)=7 \mathrm{~T}\right)$, the measured $H_{c 2}(0)$ is much smaller than expected $\left(0.7 \mathrm{~T}_{c} \partial H_{c 2} / \partial T @ \mathrm{~T}_{c}=24\right.$ and 16 $\mathrm{T}$, respectively) for purely orbital limiting [25], indicating that Pauli limiting may play an important role in determining the value of $H_{c 2}$, as in the CeMIn superconductors $[26,6] . H_{c 2}(0)$ has yet to be measured directly in PuCoGa and $\mathrm{PuRhGa}_{5}$ because of their higher $\mathrm{T}_{c}$ and correspondingly large $H_{c 2}$; however, both display anomalous behavior, with $\mathrm{PuCoGa}_{5}$ 's $H_{c 2}(T)$ being less anisotropic than expected, and PuRhGas's $H_{c 2}(T)$ displaying an anomalous shape [3].

Nuclear magnetic and quadrupole resonance (NMR and NQR) measurements on $\mathrm{Pu}-115$ superconductors provide perhaps the most direct insight into the symmetry of the superconducting order parameter in these materials (Figure 3). Knight shift measurements on $\mathrm{PuCoGa}_{5}$ reveal a spin susceptibility that decreases in the superconducting state, consistent with singlet pairing [27]. A power-law temperature dependence of the spin-lattice relaxation rate $1 / \mathrm{T}_{1} \sim \mathrm{T}^{3}$ below $\mathrm{T}_{c}$, along with absence of a Hebel-Slichter peak at $\mathrm{T}_{c}$, provides evidence for $\mathrm{d}$-wave superconductivity with line nodes in the superconducting gap [27]. Point contact spectroscopy studies of $\mathrm{PuCoGa}_{5}$ also find a four-fold modulation of the superconducting gap within the ab-plane, consistent with a $d_{x^{2}-y^{2}}$ order parameter symmetry [31]. Spin lattice relaxation measurements for $\mathrm{PuRhGa}_{5}, \mathrm{PuCoIn}_{5}$, and $\mathrm{PuRhIn}_{5}$ also find $1 / \mathrm{T}_{1} \sim$ $\mathrm{T}^{3}$ below $\mathrm{T}_{c}[28,29,30]$. For all of the $\mathrm{Pu}-115$ superconductors (Fig. 3), fits of $1 / \mathrm{T}_{1}$ to a 'dirty d-wave'model, indicate that $2 \Delta / \mathrm{k}_{B} \mathrm{~T}_{c}=5-8$. Muon spin rotation measurements have also been performed for $\mathrm{PuCoGa}_{5}$ and confirm the temperature dependences measured by NQR [32, 33]. Further, measurements of the in-plane magnetic-field penetration depth suggest d-wave pairing in $\mathrm{PuCoGa}_{5}$ and yield an estimate of zero-temperature penetration depth, $\lambda(0)$ of $241 \mathrm{~nm}[32]$. After approximately one year of aging due to self irradiation, the penetration depth in $\mathrm{PuCoGa}_{5}$ increases to $498 \mathrm{~nm}$ [33].

\section{Normal State Properties}

Because of their relatively high $\mathrm{T}_{c}$ and the general interest in the electronic structure of plutonium, a number of groups using a variety of theoretical and computational approaches have explored the electronic structure of 


\begin{tabular}{|c|c|c|c|c|c|c|c|c|}
\hline & $\begin{array}{c}T_{c} \\
(\mathrm{~K})\end{array}$ & $\begin{array}{c}\gamma_{n} \\
\left(\mathrm{~mJ} / \mathrm{mol} \mathrm{K} \mathrm{K}^{2}\right)\end{array}$ & $\begin{array}{c}H_{c 2}^{a, b} \\
(\mathrm{~T})\end{array}$ & $\begin{array}{c}H_{c 2}^{c} \\
(\mathrm{~T})\end{array}$ & $\begin{array}{c}\partial H_{c 2}^{a, b} / \partial T \\
(\mathrm{~T} / \mathrm{K})\end{array}$ & $\begin{array}{c}\partial \mathrm{H}_{c 2}^{c} / \partial T \\
(\mathrm{~T} / \mathrm{K})\end{array}$ & $\begin{array}{c}\xi_{0}^{a, b} \\
(\AA)\end{array}$ & $\begin{array}{c}\xi_{0}^{c} \\
(\AA)\end{array}$ \\
\hline $\mathrm{PuCoGa}_{5}$ & 18.5 & $\sim 80$ & $\sim 120$ & $\sim 100$ & -10 & -8 & 16 & 18 \\
\hline $\mathrm{PuRhGa}_{5}$ & 8.6 & $\sim 50$ & 31 & 17 & -3.5 & -2 & 35 & 45 \\
\hline $\mathrm{PuCoIn}_{5}$ & 2.5 & 200 & $\sim 35$ & 9.5 & -17.6 & -13.2 & 33 & 38 \\
\hline $\mathrm{PuRhIn}_{5}$ & 1.7 & 350 & $\sim 23$ & 6.5 & -20.4 & -13 & 37 & 46 \\
\hline
\end{tabular}

Table 2: Superconducting properties of plutonium superconductors. Notes: $\gamma_{n}$ : Sommerfeld coefficient at $\mathrm{T}_{c} ; H_{c 2}^{a, b}\left(H_{c 2}^{c}\right)$ : upper critical field in the $a$ - $b$ plane (parallel to the c-axis) extrapolated to $T=0 ; \partial H_{c 2} / \partial T$ : slope of the upper critical field near $T_{c} ; \xi_{0}$ : Ginzburg-Landau superconducting coherence length at $T=0$. Data from $[3,13,34]$ and references therein.

the Pu-based superconductors [35, 36, 37]. The general picture that emerges from this diversity of approaches is i) a Fermi surface that is quasi twodimensional, although not as cylindrical as the Ce-115 materials, and ii) an f-electron configuration that is partially localized and of mixed valence character. The normal state physical properties of the $\mathrm{Pu}-115$ superconductors measured experimentally are consistent with this picture.

Early photoemission spectroscopy measurements on $\mathrm{PuCoGa}_{5}$ showed that Pu's $5 \mathrm{f}$ electrons occurred in two configurations, the first being itinerant and occurring at the Fermi energy and the second being localized a little more than $1 \mathrm{eV}$ below the Fermi energy [38]. This behavior is consistent with that which has been observed in Ga-stabilized $\delta$-Pu. More recent resonant x-ray emission spectroscopy measurements on $\mathrm{PuCoGa}_{5}$ and $\mathrm{PuCoIn}_{5}$ confirm this view and further reveal an f electron configuration that includes an admixture of $5 \mathrm{f}^{5}, 5 \mathrm{f}^{4}, 5 \mathrm{f}^{6}$ states, with $\mathrm{PuCoGa}_{5}$ displaying greater mixed valence character than $\mathrm{PuCoIn}_{5}[39]$.

Significant efforts have been made to experimentally observe the Fermi surface of the $\mathrm{Pu}-115$ superconductors, especially because of the insights that have been revealed from such measurements in the Ce-115 materials [40]. Unfortunately, thesel efforts have not yet been successful, due in part to the ingrowth of irradiation damage that begins upon initial solidification of the material, shortening the time available to conduct such measurements before disorder obscures the desired quantum oscillations.

Consistent with this picture of the f-electron configuration of the $\mathrm{Pu}-115$ materials, the electrical resistivity of $\mathrm{PuCoGa}_{5}$ is weakly temperature dependent at high temperature before developing a curvature consistent with mixed 


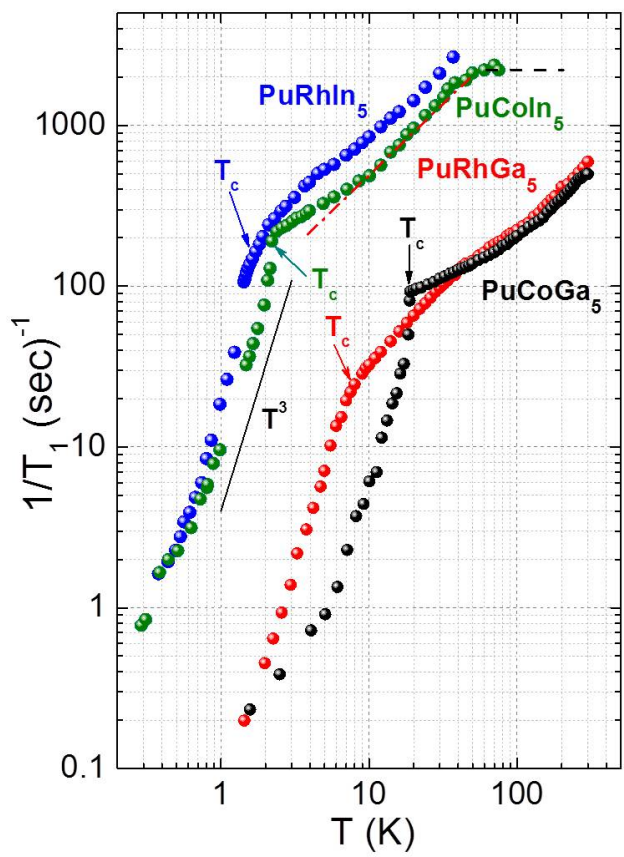

Figure 3: Spin-lattice relaxation rate $1 / \mathrm{T}_{1}$ versus $\mathrm{T}$ for each of the known plutonium superconductors. The solid black line is a $\mathrm{T}^{3}$ power law, reflective of the observed behavior below $\mathrm{T}_{c}$. The normal state behavior is approximately Korringa-like at intermediate temperatures above $\mathrm{T}_{c}$.

valence materials below $150 \mathrm{~K}$. At lowest temperatures, $\rho(T)$ assumes an unusual power-law T-dependence: $\rho \sim T^{4 / 3}[1]$ that also appears in $\mathrm{PuRhGa}_{5}$ [11]. $\rho(T)$ of $\mathrm{PuCoIn}_{5}$ and $\mathrm{PuRhIn}_{5}$ is nearly temperature independent above $150 \mathrm{~K}$ before passing through a maximum around $100 \mathrm{~K}$ and then decreasing rapidly at lower temperatures, again consistent with mixed valence or Kondo lattice behavior [3]. At lowest temperature, $\rho(T) \sim T^{n}$ with $\mathrm{n}=1$ and $4 / 3$ for $\mathrm{PuRhIn}_{5}$ and $\mathrm{PuCoIn}_{5}$, respectively, power-laws that are again distinctly different from the expected $T^{2}$ Fermi liquid dependence. Insufficient data exist to make strong statements about the implied non-Fermi liquid behavior in all of the Pu-115 superconductors. However, such behavior often occurs in nearly-magnetic materials, as these plutonium materials appear to be, given their normal state electronic configuration and potentially d-wave superconducting state. 
Bulk magnetic susceptibility has been measured in $\mathrm{PuRhIn}_{5}, \mathrm{PuRhGa}_{5}$, and $\mathrm{PuCoGa}_{5}$. In $\mathrm{PuRhIn}_{5}, \chi(T)$ follows a modified Curie-Weiss behavior above $100 \mathrm{~K}$ with an effective moment $\mu_{\text {eff }}=0.85 \mu_{B}\left(0.3 \mu_{B}\right)$ and an antiferromagnetic Weiss temperature $\Theta=-120 \mathrm{~K}(-7 \mathrm{~K})$ for a magnetic field perpendicular (parallel) to the c-axis [3]. In contrast to $\mathrm{PuRhIn}_{5}, \chi(T)$ is only weakly anisotropic in $\mathrm{PuRhGa}_{5}[9]$ despite a Curie-Weiss behavior that is consistent with a $\mathrm{Pu}^{3+}$ configuration. In $\mathrm{PuCoGa}_{5}$ polarized neutron measurements of the microscopic magnetization reveal a small and weakly temperature dependent magnetic moment [41], in contrast to initial bulk measurements that revealed Curie-Weiss behavior [1], perhaps consistent with the mixed valence configuration inferred from x-ray emission spectroscopy.

NMR and NQR measurements have also been performed in the normal state of each of the $\mathrm{Pu}-115$ superconductors (Figure 3). $\mathrm{In} \mathrm{PuCoIn}_{5} 1 / \mathrm{T}_{1}$ is essentially temperature independent above $60 \mathrm{~K}$, indicating the presence of weakly coupled $5 \mathrm{f}$ magnetic moments and anticipating that the bulk magnetic susceptibility will display Curie-Weiss behavior as in $\mathrm{PuRhIn}_{5}$ [29]. Between 10 to $40 \mathrm{~K}$, Korringa-like relaxation, $1 / \mathrm{T}_{1} \sim \mathrm{T}$, appears and is followed at lower temperatures by a faster relaxation rate, perhaps due to the presence of antiferromagnetic spin fluctuations. This scenario could also explain the anomalous low-temperature behavior of the electrical resistivity discussed above. $\mathrm{PuRhIn}_{5}$ spin-lattice relaxation behavior is similar to that of $\mathrm{PuCoIn}_{5}$ [30]. 1/ $\mathrm{T}_{1}$ in $\mathrm{PuCoGa}_{5}$ and $\mathrm{PuRhGa}_{5}$ are similar in temperature dependence and notably slower in magnitude than their In-based counterparts. Below $\sim 30 \mathrm{~K}, \mathrm{PuCoGa}_{5}$ displays a temperature dependence that is decidedly not Korringa-like [27].

\section{Perspective}

By most accounts, the superconductivity observed in the $\mathrm{Pu}-115$ materials is not conventional, i.e., an explanation of observed properties in terms of phonon-mediated pairing consistent with the BCS theory is incomplete. As discussed above, the temperature dependence of the spin-lattice relaxation rate and the magnetic penetration depth below $\mathrm{T}_{c}$ yield power-law behavior consistent with d-wave pairing. Further, point-contact spectroscopy reveals a four-fold modulation of the superconducting gap. However, relative to e.g., the high- $\mathrm{T}_{c}$ cuprates, the direct evidence of d-wave superconductivity is rather limited. 
In the case of the Ce-115 materials, the evidence for d-wave superconductivity is quite strong, and the numerous similarities between the Ce-115 and $\mathrm{Pu}-115$ materials at least suggest a similar origin of superconductivity. In both the Ce-115 and Pu-115 materials, a universal structural tuning is found in which $\mathrm{T}_{c}$ varies linearly with the $\mathrm{c} / \mathrm{a}$, the ratio of the tetragonal lattice constants, even in heavily alloyed materials in which the naive expectation would be that superconductivity is destroyed [17]. Further, the individual $\mathrm{Pu}-115$ superconductors can be mapped, based on their unit cell volumes, onto a generalized Doniach phase diagram that is analogous to that observed in Ce-115 and suggests that the superconducting compounds exist in close proximity to magnetic order [12]. This proximity to magnetism is also often used to explain the unit cell volume of elemental plutonium

If one assumes that the superconductivity is mediated by spin fluctuations, a comparison can be made between superconducting $\mathrm{T}_{c}$ and a characteristic spin fluctuation temperature $\mathrm{T}_{s f}$ inferred from normal state properties $[27,44]$. In this scenario, a linear relationship between $\mathrm{T}_{c}$ and $\mathrm{T}_{s f}$ is observed among the plutonium superconductors, but more dramatically (Figure 4), the $\mathrm{Pu}-115$ materials appear to bridge a gap in $\mathrm{T}_{c}$ and $\mathrm{T}_{s f}$ between more traditional heavy fermion superconductors and the cuprates.

While the strong analogies with the Ce-115 materials and the overall correlation with other heavy fermions and cuprates are suggestive, it would seem premature to conclude that superconductivity in the $\mathrm{Pu}-115$ materials is mediated solely by spin fluctuations. Similarly, one should not assume that all of the $\mathrm{Pu}-115$ materials have the same pairing mechanism given that, e.g., $\mathrm{PuCoIn}_{5}$ and $\mathrm{PuCoGa}_{5}$ have rather different normal state magnetic susceptibilities. In particular, the fact that $\mathrm{Pu}$ has multiple $5 \mathrm{f}$ electrons and they appear to exist in an admixture of $5 \mathrm{f}$ states suggests that more complex valence fluctuations might be responsible for mediating superconductivity $[39,42]$. Theoretical work is ongoing to explore such scenarios, including critical valence fluctuations and/or density fluctuations as the origins of pairing $[44,43,45,46,47]$. In these scenarios, the characteristic temperature in Figure 4 may be more general than a spin fluctuation scale and characterize a Kondo or valence fluctuation energy. A full exploration of these theoretical approaches and their normal- and superconducting-state signatures might provide insight not only to plutonium superconductors but also to broader classes of strongly correlated materials. 


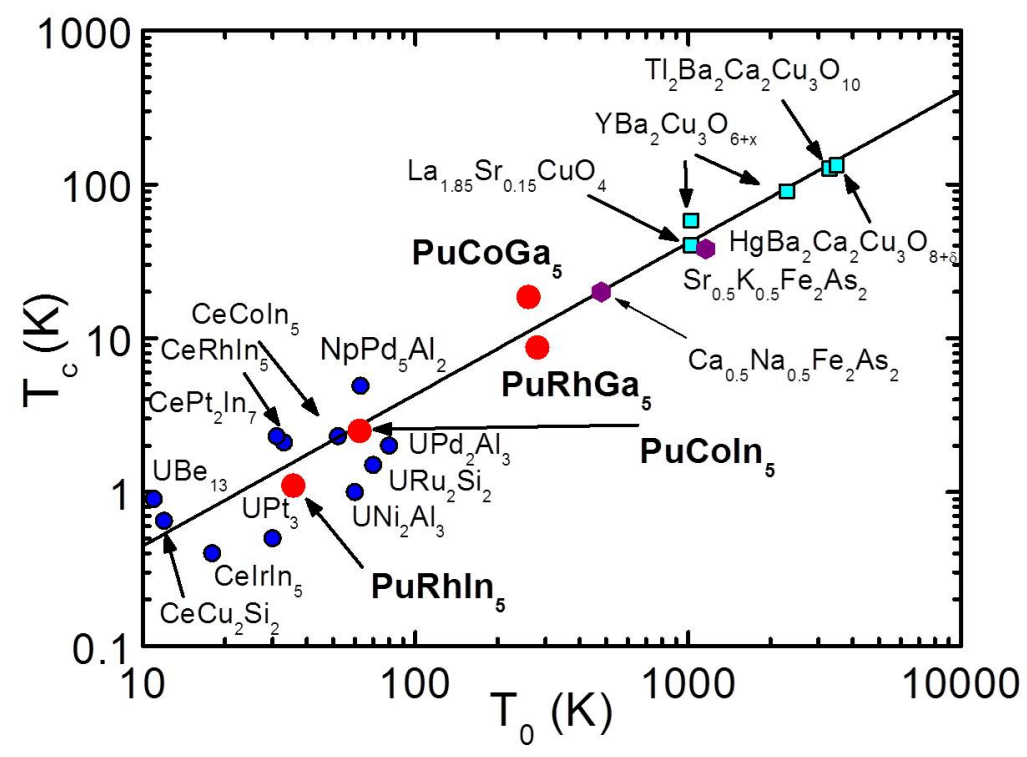

Figure 4: Superconducting transition temperature $\mathrm{T}_{c}$ versus a characteristic temperature $\mathrm{T}_{0}$ for several families of superconductors. The plutonium superconductors occupy a region of $T_{c}$ and $T_{0}$ that are intermediate between heavy fermion superconductors and high- $\mathrm{T}_{c}$ cuprates.

\section{Conclusion}

The superconductivity that has been observed in the $\mathrm{Pu}-115$ family of compounds raises as many questions as it answers. Qualitatively, the physical properties of these materials display many attributes that are consistent with other strongly correlated superconductors and also embody many of the mysteries of elemental plutonium and its allotropes. A scenario in which unconventional superconductivity emerges from a strongly-correlated mixed valent normal state at relatively high temperature given measured fluctuation energies is plausible. However, quantitatively, much more work remains to definitively prove a pairing mechanism, e.g., via a full suite of phase-sensitive measurements, in these materials. Further, the phase space of plutonium intermetallic compounds has only just begun to be explored. While only four plutonium-based superconductors are known, they represent a larger fraction of all known $\mathrm{Pu}$-based intermetallic compounds than e.g., in transition metal or rare earth compounds. Fundamentally, this should leave one optimistic 
that further discoveries await that will increase the number of known unconventional superconductors and clarify the enigma that is the electronic and metallic properties of elemental plutonium.

\section{Acknowledgments}

Work at Los Alamos National Laboratory was performed under the auspices of the U.S. Department of Energy and was supported by the Office of Basic Energy Sciences, Division of Materials Science and Engineering as well as the Los Alamos National Laboratory Laboratory Directed Research and Development (LDRD) program.

\section{References}

[1] J.L. Sarrao, L.A. Morales, J.D. Thompson, B.L. Scott, G.R. Stewart, et al., Nature 420, 297 (2002).

[2] J.L. Sarrao and J.D. Thompson, J. Phys. Soc. Jpn. 76, 051013 (2007).

[3] E.D. Bauer and J.D. Thompson, to appear in Annual Review of Condens. Matter Physics 6 (2015).

[4] J.L. Sarrao, L.A. Morales, and J.D. Thompson, JOM 55, 38 (2003).

[5] E.G. Moshopoulou, Z. Fisk, J.L. Sarrao, and J.D. Thompson, J. Solid State Chem. 158, 25 (2001).

[6] J.D. Thompson and Z. Fisk, J. Phys. Soc. Jpn. 81, 011002 (2012).

[7] E.D. Bauer, H.O. Lee, V.A. Sidorov, N. Kurita, K. Gofryk, et al., Phys Rev B 81, 180507 (2012).

[8] M. Nicklas, V.A. Sidorov, H.A. Borges, P.G. Pagliuso, et al., Phys. Rev. B 67, 020506 (2003).

[9] P. Boulet, E. Colineau, F. Wastin, P. Javorsky, J.C. Griveau, et al., Phys. Rev. B 72, 064438 (2005).

[10] J.L. Sarrao, J.D. Thompson, N.O. Moreno, L.A. Morales, F. Wastin, et al., J. Phys.: Condens. Matter 15, S2275 (2003). 
[11] F. Wastin, P. Boulet, J. Rebizant, E. Colineau, and G.H. Lander, J. Phys. Condens. Matter 15, S2279 (2003).

[12] E.D. Bauer, M.M. Altarawneh, P.H. Tobash, K. Gofryk, O.E. AyalaValenzuela, J. Phys. Condens. Matter 24052206 (2012).

[13] E.D. Bauer, P.H. Tobash, J.N. Mitchell, J.L. Sarrao, Phil. Mag. 92, 2466 (2012).

[14] S.S. Hecker, MRS Bulletin 26, 672 (2001).

[15] S.Y. Savrasov, G. Kotliar and E. Abrahams, Nature 410, 793 (2001).

[16] J.L. Smith, Z. Fisk and S.S. Hecker, Physica B \& C 130, 151 (1985).

[17] E.D. Bauer, J.D. Thompson, J.L. Sarrao, L.A. Morales, F. Wastin, et al., Phys. Rev. Lett. 93147005 (2004).

[18] P. Javorsky, E. Colineau, F. Wastin, F. Jutier, J.C. Griveau, et al.,Phys. Rev. B 75,184501 (2007).

[19] C.H. Booth, E.D. Bauer, M. Daniel, R.E. Wilson, J.N. Mitchell, et al., Phys. Rev. B 76, 064530 (2007).

[20] F. Jutier, J.C. Griveau, E. Colineau, J. Rebizant, P. Boulet, et al., Physica B 359, 1078 (2005).

[21] F. Jutier, G.A. Ummarino, J.C. Griveau, F. Wastin, E. Colineau, et al., Phys. Rev. B 77, 024521 (2008).

[22] D. Talbayev, K.S. Burch, E.E.M. Chia, S.A. Trugman, J.-X. Zhu, et al., Phys. Rev. Lett. 104, 227002 (2010).

[23] Y. Haga, D. Aoki, T. Matsuda, K. Nakajima, Y. Arai, et al., J. Phys. Soc. Jpn. 74, 1698 (2005).

[24] E.D. Bauer, T. Park, R.D. McDonald, M.J. Graf, L.N. Boulaevskii, et al., J. Alloys Cmpds. 488, 554 (2009).

[25] N.R. Werthamer, E. Helfand, and P.C. Hohenberg, Phys. Rev. 147, 295 (1966). 
[26] A. Bianchi, R. Movshovich, C. Capan, P.G. Pagliuso, and J.L. Sarrao, Phys. Rev. Lett. 91, 187004 (2003).

[27] N.J. Curro, T. Caldwell, E.D. Bauer, L.A. Morales, M.J. Graf, et al., Nature 434, 622 (2005).

[28] H. Sakai, Y.Tokunaga, T. Fujimoto, S.E. Kambe, R. Walstedt, et al., J. Phys. Soc. Jpn. 74, 1710 (2005).

[29] G. Koutroulakis, H. Yasuoka, H. Chudo, P.H. Tobash, J.N. Mitchell, et al., New J. Phys. 16, 053019 (2014).

[30] G. Koutroulakis, A.M. Mounce, M. Wartenbe, N.Ghimire, H. Yasouka, et al., unpublished.

[31] D. Daghero, M. Tortello, G.A. Ummarino, J.C. Griveau, E. Colineau, et al., Nat. Comm. 3, 786 (2012).

[32] G.D. Morris, R.H. Heffner, E.D. Bauer, L.A. Morales, J.L. Sarrao, et al., Physica B 374, 180 (2006).

[33] K. Ohishi, R.H. Heffner, G.D. Morris, E.D. Bauer, M.J. Graf, et al., Phys. Rev. B 76, 064504 (2007).

[34] J.C. Griveau and E. Colineau, C. R. Physique 15, 599 (2014).

[35] T. Das, J.X. Zhu, and M.J. Graf, J. Mater. Res. 28, 659 (2013).

[36] I. Opahle, S. Elgazzar, K. Koepernik, and P.M. Oppeneer, Phys. Rev. B 70, 104504 (2004).

[37] T. Maehira, T. Hotta, K. Ueda, and A. Hasegawa, Phys. Rev. Lett. 90, 207007 (2003).

[38] J.J. Joyce, J.M. Wills, T. Durakiewicz, M.T. Butterfield, E. Guziewicz, et al., Phys. Rev. Lett. 91, 176401 (2003).

[39] C.H. Booth, S.A. Medling, Y. Jiang, E.D. Bauer, P.H. Tobash, et al., J. Electr. Spec. Rel. Phenomen. 194, 57 (2014).

[40] H. Shishido, R. Settai, D. Aoki, S. Ikdea, H. Nakawaki, et al., J. Phys. Soc. Jpn. 71, 162 (2002). 
[41] A. Hiess A. Stunault, E. Colineau, J. Rebizant, F. Wastin, et al., Phys. Rev. Lett. 100, 076403 (2008).

[42] B.J. Ramshaw, A. Shekhter, R.D. McDonald, J.B. Betts, J.N. Mitchell, et al., arXiv:1409.4118v1 (2014).

[43] K. Miyake, J. Phys. Condens. Matter 19, 125201 (2007).

[44] P. Monthoux, D. Pines and G.G. Lonzarich, Nature 450, 1177 (2007).

[45] R. Flint, M. Dzero, and P. Coleman, Nature Physics 4, 643 (2008)

[46] A.B. Shick, J. Kolorenc, J. Rusz, P.M. Oppeneer, A.I. Lichtenstein, et al., Phys. Rev. B 87, 020505 (2013).

[47] A.B. Shick and J. Kolorenc, C. R. Physique 15, 640 (2014). 